\title{
Ethnographe, archiviste, producteur, activiste... Les nombreuses vies d'Anthony Seeger
}

Jonathan P. J. Stock et Anthony Seeger

Traducteur : Ramèche Goharian

\section{OpenEdition}

\section{Journals}

Édition électronique

URL : http://journals.openedition.org/ethnomusicologie/120

ISSN : 2235-7688

Éditeur

ADEM - Ateliers d'ethnomusicologie

Édition imprimée

Date de publication : 1 novembre 2006

Pagination : 221-250

ISSN : 1662-372X

\section{Référence électronique}

Jonathan P. J. Stock et Anthony Seeger, «Ethnographe, archiviste, producteur, activiste... Les nombreuses vies d'Anthony Seeger », Cahiers d'ethnomusicologie [En ligne], 19 | 2006, mis en ligne le 15 janvier 2012, consulté le 22 avril 2019. URL : http://journals.openedition.org/ethnomusicologie/120

Ce document a été généré automatiquement le 22 avril 2019

Tous droits réservés 


\title{
Ethnographe, archiviste, producteur, activiste... Les nombreuses vies d'Anthony Seeger ${ }^{1}$
}

\author{
Jonathan P. J. Stock et Anthony Seeger \\ Traduction : Ramèche Goharian
}

1 Plus qu'aucun autre ethnomusicologue, Anthony Seeger semble avoir débordé d'activités pendant ses soixante ans de vie. Actuellement professeur à l'Université de Californie, Los Angeles, il a occupé des postes académiques importants au Brésil et aux Etats-Unis, a été directeur des Archives de Musique Traditionnelle à l'Université d'Indiana, conservateur et directeur au Smithsonian Folkways Records à Washington DC, puis président et secrétaire général du Conseil international de la musique traditionnelle (International Council for Traditional Music, ICTM). Ses publications sont appréciées pour la profondeur de leur analyse musicale et sociale, pour l'originalité de leur approche théorique et méthodologique, pour la saveur de leur style lumineux, ainsi que pour leur simplicité et leur chaleur humaine.

Dans cet entretien, Anthony Seeger médite sur son éducation familiale en tant que petitfils du philosophe de la musique et activiste, Charles Seeger. Par moments, la carrière personnelle d'Anthony suit à la trace celle de son grand-père en une sorte d'écho à la fois décalé et sympathique. L'ethnomusicologue esquisse ensuite ses expériences intellectuelles et musicales dans l'Amérique maccarthyste avant d'aborder l'influence de la pratique musicale dans les communitas de l'université et de parler de son mariage. Il expose les idées théoriques qui le menèrent tout d'abord chez les Suyá du Brésil et explique la réalité de la vie et de la recherche sur le terrain dans une société sans argent, et dont il n'a pas préalablement appris la langue. Cette longue période de contact ainsi que ses visites ultérieures furent à l'origine de nouvelles découvertes et de l'établissement de relations durables. Aujourd'hui encore, cette expérience continue de modeler certains aspects de sa démarche. 
Seeger décrit ensuite le cheminement de sa carrière qui le conduisit de Rio de Janeiro à Bloomington, Indiana, puis de Washington DC à Los Angeles, le faisant passer d'un poste académique à la direction d'archives, puis le ramenant de la production de disques à l'enseignement. Ses réponses révèlent la continuité remarquable qui sous-tend ce cheminement. Après avoir mentionné son rôle au sein de l'International Council for Traditional Music, il conclut par quelques réflexions sur les projets passés ou futurs qui lui tiennent à cœur. Une bibliographie sélective est proposée en annexe.

\section{J. S.}

Fig 1 : Tony Seeger, portrait.

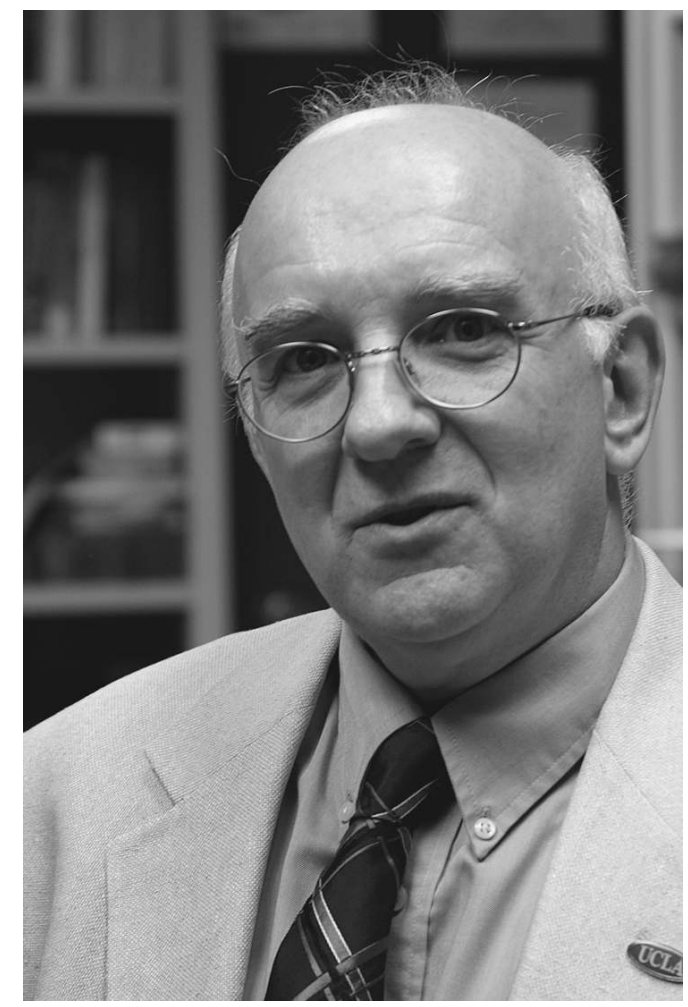

Photo : Roger Bourland, 2005

Pourriez-vous décrire en quelques mots votre environnement familial et expliquer de quelle manière il vous a poussé vers la carrière que vous avez embrassée, et comment il a influencé la façon particulière que vous avez choisie pour mener à bien cette carrière?

Je suis né le 29 mai 1945 à New York, dans le quartier de Manhattan. Comme je suis né dans une famille très musicienne, ma vie allait inévitablement tourner d'une façon ou d'une autre autour de la musique. Mon grand-père, Charles Seeger, avait sept enfants de deux mariages. Ma grand-mère, Constance Seeger, était sa première femme. Violoniste concertiste, elle l'épousa lorsqu'il était un jeune compositeur et ils eurent trois enfants : Charles Jr., John (mon père) et Pete. Mon père se souvient de l'aventure où la famille "amenait la musique au peuple " dans une remorque bricolée, tirée par une Ford Modèle A. Il se voit encore, assis sur le siège arrière de la voiture, en train de chanter des chansons en harmonie avec ses frères. Plus tard Charles épousa la compositrice Ruth Crawford et ils eurent quatre enfants : Mike, Peggy, Barbara et Penny. Lorsque j'étais enfant, Pete, Mike et Peggy Seeger étaient des musiciens très actifs. Ils enregistraient chez Folkways Records (une petite maison de disque 
indépendante basée à New York, fondée par Moses Asch) ${ }^{2}$ et m'offraient des disques Folkways à Noël.

J'ai passé les six premières années de ma vie à Greenwich Village, tout près de Washington Square. Je roulais en tricycle autour du square et écoutais des concerts de fanfares à la Saint-Sylvestre. Greenwich Village était le quartier des artistes et j'y ai rencontré des musiciens dès mon plus jeune âge. C'est là que j'ai vu Leadbelly, un soir de Noël, alors que j'étais encore dans mon berceau ; j'ai rencontré Woody Guthrie à un concert à l'âge de six ans ; j'ai assisté à des concerts de Pete Seeger, des Weavers, des New Lost City Ramblers et d'autres au Carnegie Hall, au Town Hall etc.

Mes parents s'étaient rencontrés dans une école et un camp où ils avaient chanté ensemble, ils tombèrent amoureux et finirent par se marier. Ils continuèrent à chanter ensemble a cappella tout au long de leur vie. Je me rappelle - et j'en suis un peu honteux - d'un jour où je me trouvais assis entre eux sur le siège de la voiture (il n'y avait pas de ceinture de sécurité à l'époque). Je leur ai demandé de se taire parce que j'allais chanter. Hélas, ils se turent, et moi, je n'ai jamais cessé de chanter depuis !

Mes parents étaient instituteurs et enseignaient dans une école privée; j'ai toujours fréquenté de très bonnes écoles progressistes qui offraient, à un niveau supérieur à la moyenne, la possibilité d'accéder à l'enseignement et à l'interprétation de la musique. A l'école primaire, tous les enseignants me connaissaient du fait que mes parents $y$ travaillaient, et j'ai sans doute bénéficié d'un traitement de faveur. Vers neuf ans je commençai à jouer de la flûte et, peu après, je me mis au violon. Je suis gaucher et, de toute façon, je n'ai jamais été très à l'aise avec l'archet. Ma grand-mère, Constance, quittait chaque année la Floride pour fuir les ouragans et elle venait passer une partie de ses visites annuelles chez nous. Elle profitait de ces occasions pour m'enseigner le violon, que je n'arrivais pas vraiment à apprécier. Mon jeu de violon se dégradant, je commençai, vers l'âge de onze ans, à jouer du banjo.

En plus de leur métier d'enseignants, mes parents organisaient dans leur propriété du Vermont un camp d'été pour les enfants. Ils l'avaient repris en 1949, quand j'avais quatre ans. J'y passai plus de vingt-cinq étés de ma vie. C'est là que j'appris successivement le banjo, la guitare, l'autoharp et d'autres instruments. J'étudiais auprès d'Ed Badeaux, un musicien folk texan qui me donnait des cours, en hiver comme en été, au camp. Bientôt je commençai à donner des concerts à l'école; en huitième, j'avais 150 chansons à mon actif et un fan club de filles des plus petites classes. Dès l'école primaire, mon identité fut, dans une certaine mesure, associée à ma capacité de chanter et de jouer du banjo. Non pas que j'étais particulièrement doué ; comme beaucoup d'autres membres de ma famille, j'étais timide dans la vie privée, mais une fois monté sur scène, je devenais vraiment intrépide (pour ne pas dire sans gêne).

En tant qu'enfant ayant grandi dans la famille Seeger pendant les années 1950, j'étais convaincu que la musique était plus qu'un simple divertissement. C'était dû à l'impact des mouvements anticommunistes largement répandus aux Etats-Unis. En 1951, les Weavers - dont faisait partie mon oncle Pete - avaient des chansons au Hit Parade de la musique populaire américaine. En 1953, ils furent dispersés car ils figuraient sur la « liste noire ». Cette même année, alors que j'écoutais un disque 78 tours où mon oncle chantait « Talking Union » par une journée chaude et étouffante à New York, mon père, d'habitude si placide, se rua dans la chambre, ferma brusquement les fenêtres et, furieux, me dit de ne plus jamais écouter ce disque les fenêtres ouvertes. Il avait 
manifestement eu peur. Son frère aîné, Charles, avait dû émigrer à Leiden pour trouver du travail à cause de la liste noire. Son père Charles ne pouvait plus obtenir de passeport pour voyager et s'était retiré prématurément de son poste au Pan American Union. Ce n'était pas vraiment le moment que les voisins entendent des chansons syndicalistes sortant de nos fenêtres.

Pete fut convoqué pour témoigner devant la Commission sur les Activités antiaméricaines. Il plaida le Premier amendement et invoqua la liberté d'association; il dut comparaître pour outrage au Congrès. Ses démêlés juridiques continuèrent tout au long des années 1950 et j'étais au courant de tout cela. Lorsqu'il proposa de chanter devant le juge les chansons qu'il avait chantées pendant les années 1940 devant des groupes prétendument subversifs, le juge refusa de l'écouter. Pete réagit en les chantant debout sur les marches du tribunal devant un public enthousiaste. Il ne passa pas une seule journée en prison; mais sa carrière - ainsi que celle de centaines d'autres - fut profondément bouleversée par la peur et la haine dirigées contre lui, ainsi que par le soutien de ses admirateurs qui continuèrent à fredonner ses chansons. Le point positif dans tout cela fut l'achat de notre premier téléviseur ; en effet, mes parents qui avaient toujours refusé d'en avoir un, voulurent suivre en direct le procès intenté contre McCarthy.

Je pense que c'est en partie l'expérience de ces années qui m'incita à devenir ethnomusicologue ou, du moins, qui éveilla mon intérêt. J'étais persuadé que la musique ne servait pas uniquement à divertir. Si elle n'avait pas de pouvoir, les gens qui en jouaient ne seraient pas mis à l'index ou en prison. J'appris tout seul de nombreuses chansons protestataires et en composai même quelques unes. Ma première expérience d'une musique non occidentale vint plus tard. Je crois qu'elle remonte à une composition que je fis quand j'avais douze ans. Nous consacrions une partie de l'année à étudier l'histoire et la culture de l'Inde. Chaque élève devait écrire un texte sur un aspect de la civilisation de ce pays. Je décidai d'écrire le mien sur la musique. Je découvris un disque Folkways sur la musique classique et populaire de l'Inde (remarquez le nombre de fois que ce label entre dans ma vie). Je le rapportai à la maison, lus la pochette et l'écoutai. Une bonne partie du disque ne me toucha pas vraiment mais une plage d'un raga pour shenai et tabla me ravit : je l'adorais. Elle ne durait pas plus de cinq minutes, mais certaines structures du morceau me paraissaient évidentes et je l'écoutais en boucle. Ma mère l'appelait improprement « la torture chinoise de la goutte d'eau », ce qui me rendait la chose encore plus aimable. J'écrivis ma dissertation et elle fut très bien notée. Deux ans plus tard, à quatorze ans, j'achetai un coffret de disques Folkways, «African Music South of the Sahara ", compilés et annotés par Alan Merriam. J'écrivis à nouveau un texte, cette fois-ci pour ma classe de musique, qui fut, lui aussi, très bien noté. L'année suivante, je composai un essai sur la musique japonaise et j'achetai à cet effet un autre disque Folkways ainsi qu'un livre incroyablement beau et intéressant de William P. Malm, ma première monographie ethnomusicologique.

J'ai commencé l'ethnomusicologie plus tôt que la plupart de mes collègues, d'une part parce que j'ai grandi dans la famille Seeger, et d'autre part parce que j'avais à portée de main l'extraordinaire collection des musiques éditées par la maison de disques indépendante de New York, Folkways Records. Les découvertes musicales de mon enfance furent si puissantes que lorsqu'on me proposa, quelques décennies plus tard, le poste de conservateur et de directeur de Folkways Records à la Smithsonian Institution, je 
l'acceptai sachant combien les sons enregistrés peuvent être utiles et inspirants pour les gens.

Où, quand et avec qui avez-vous étudié la musique ? A quoi attribuez vous ces choix ?

Pendant mes années de lycée, entre 1959 et 1963, je fus interne à la Putney School, une école mixte située dans les collines du Sud Vermont. Les élèves n'avaient pas le droit d'apporter d'équipement de reproduction sonore à l'école - les casques n'existaient pratiquement pas à l'époque - et nous devions jouer nous mêmes toute la musique que nous écoutions. Les seules exceptions étant quelques 33 tours qu'on nous passait dans les classes de musique et la possibilité d'écouter nos propres disques, à certaines heures du week-end, dans une cabine froide et lugubre qui était souvent occupée par des couples recherchant la solitude. Je jouais le banjo et la guitare pendant des heures interminables et je chantais dans le chœur, mais j'abandonnai le violon. Le professeur de musique et chef d'orchestre Norwood Hinkle dirigeait non seulement l'orchestre de l'école, mais, tous les vendredis, il faisait également chanter un chœur de madrigalistes composé d'étudiants et de membres du personnel de l'école. En outre, il enseignait l'histoire de la musique européenne et le contrepoint élémentaire. L'enseignement qu'il prodigua pendant mes années de lycée constitue la seule forme d'instruction musicale que j'ai reçue dans ces domaines.

Tout en prenant mes études au sérieux, je faisais de longues promenades à cheval dans les collines, au printemps et en automne, et je skiais en hiver. Tous mes professeurs écrivaient sur mon carnet « Tony serait un étudiant formidable s'il se donnait un peu plus de peine ». Je fus admis à l'Université de Harvard en 1963, probablement, grâce à ma réputation de joueur de banjo au lycée. Ce ne fut en tout cas pas à cause de mes notes, qui n'étaient pas fameuses.

A Harvard, je crus utile de m'inscrire à des cours de musique. Mais je fus outré d'apprendre qu'avant de pouvoir le faire, je devais d'abord suivre un cours élémentaire d'instrument à clavier pour les exercices d'harmonie. Je pensais qu'on devait m'accorder l'autorisation d'utiliser ma guitare, comme au lycée, et je refusai de me plier aux exigences de l'université. Je ne suivis donc aucun cours de musique. Ma famille ne m'encourageait d'ailleurs pas du tout dans ce sens. Mon grand-père Charles avait dit à mon père «Eloigne Tony du département de musique : ils vont l'abîmer. » Le département de musique n'eut jamais cette occasion.

A l'université, je n'ai pratiquement pas étudié. Je connaissais depuis longtemps Judy Austin qui étudiait à l'école supérieure des jeunes filles affiliée à Harvard. Nous avons commencé à chanter ensemble à l'université, à passer des week-ends au Vermont et nous nous sommes fiancés. Au lieu d'assister à la remise des diplômes à Harvard, nous nous sommes mariés et embarqués ensemble vers des études de troisième cycle.

Qui a le plus influencé votre réflexion lorsque vous étiez un apprenti anthropologue, pourquoi et comment?

Ma branche principale de licence me permit d'acquérir de bonnes connaissances en sciences sociales (sociologie, anthropologie sociale et psychologie sociale). Je fus très influencé par les cours des sociologues Talcott Parsons et Robert Bellah et des anthopologues David Maybury-Lewis et Evon Z. Vogt. J'ai aussi eu d'excellents doctorants tuteurs comme Victor Lidz et Pierre Maranda. Je combinai les sciences sociales avec l'étude du folklore. Je pris plusieurs cours avec Albert Lord (auteur de The Singer of Tales), qui rêvait de me voir devenir spécialiste de l'épopée. Parmi les livres 
d'anthropologie qui me marquèrent, je citerais Tristes tropiques et Le cru et le cuit de Claude Lévi-Strauss. Dans une première tentative d'analyses informatiques du mythe, je créai péniblement un « dictionnaire des opposés » que je tapais sur les cartes perforées IBM. J'écrivis mon mémoire sur les peuples de l'Arnhem Land en Australie et les sociétés septentrionales de langue Gê du Brésil - qui présentent, croyez-moi, des similarités très intéressantes. En dernière année de licence, il me semblait que c'était l'anthropologie qui posait les questions les plus intéressantes; je choisis donc de continuer mes études de troisième cycle dans cette branche. Quant à Judy, elle décida d'étudier l'espagnol, le portugais et la littérature.

Avec Judy, nous posâmes nos candidatures dans les mêmes cinq universités et choisîmes celle qui nous accordait le meilleur soutien financier, l'Université Cornell. Nous avions de la chance. Je souhaitais entrer à Cornell pour étudier avec Victor Turner, dont l'œuvre était ce que j'avais lu de plus intéressant sur le symbolisme rituel. Je voulais également étudier avec Terence S. Turner (aucun rapport avec Victor Turner), un étudiant de David Maybury-Lewis, spécialiste de l'organisation sociale et de la cosmologie des Indiens Kayapó du Brésil. Judy étudiait dans le département d'espagnol et de portugais. "Vic» et "Terry» déménagèrent à Chicago l'année suivante, en 1968. Judy et moi les suivîmes, et nous finîmes donc nos études de doctorat à l'Université de Chicago.

Mes professeurs Victor et Terence Turner influencèrent profondément ma vie et mes recherches. Tous les mardis soir, Victor organisait chez lui un séminaire auquel j'ai participé pendant quatre ans. En plus de la lecture de ses propres écrits, Vic invitait de nombreux collègues à ces soirées, dont l'anthropologue brésilien Roberto Da Matta qui jouera plus tard un rôle très important dans ma carrière brésilienne. Le fait de nous rencontrer chez Victor Turner créa un lien durable entre nous. Victor ne faisait pas qu'écrire sur les communitas, il y croyait profondément. Il aimait vraiment la musique, les fêtes, l'absence de hiérarchie et de structure. Nous avons passé tant de soirées chez lui à jouer du banjo et de la guitare, à chanter en groupe avec tout le monde! Terry Turner avait rapporté de son année passée en Angleterre un énorme répertoire de chants de rugby ; ajoutés à d'autres répertoires, cela prolongeait nos réunions jusque tard dans la nuit. Ces soirées, et le fait de vivre réellement ses théories eurent un profond impact sur moi en tant qu'individu et que scientifique. J'appris énormément de mes professeurs et de mes collègues étudiants à l'Université de Chicago, où je passai trois années très agréables.

A cette époque, il n'y avait pas encore d'ethnomusicologie à l'Université de Chicago. Le chef du département de musique me proposa de donner un cours d'ethnomusicologie avec Ella Zonis, dont le mari enseignait au programme d'études sur le Moyen-Orient. Elle venait récemment de rentrer d'Iran où elle avait étudié la musique classique persane. Jusqu'ici, le fait de ne pas connaître à fond un domaine ne m'avait pas dérangé, mais je me mis à lire tout ce que je pus sur le sujet. Je m'occupais d'anthropologie et Ella d'analyse musicale plus traditionnelle. Nous eûmes beaucoup de plaisir à enseigner ensemble ce printemps-là, après quoi je partis faire des recherches au Brésil.

Il y a différentes façons d'aborder la musique. Il est évident que l'approche et les méthodes employées par chaque scientifique influencent ses résultats. Je me suis toujours intéressé à la musique en tant qu'activiste social (comment pouvons-nous remuer le peuple avec la musique, et pourquoi ?) et qu'anthropologue (comment la 
musique participe-t-elle aux processus sociaux, au langage et à la pensée ?). Il y a quelques années, à la conférence mondiale de l'ICTM à Rio de Janeiro, j'ai présenté une allégorie de l'étude de la musique avec une banane et un couteau pointu (Seeger 2003). Une banane paraît différente lorsque elle est coupée différemment; de même, différentes approches de la musique donnent des résultats différents - pourtant il s'agit toujours du même objet. Toutes les approches sont plus ou moins valables, mais seules certaines intéressent une personne en particulier. Je ne fais aucun mystère de mes intérêts et de mon attirance pour l'ethnomusicologie ; ils découlent de mon éducation, de mes expériences d'auditeur et d'interprète ainsi que de ma formation en sciences sociales.

Beaucoup d'entre nous vous connaissent par vos écrits sur les Suyá. Pourquoi eux, et pourquoi vous?

Les gens me demandent souvent pourquoi j'ai choisi d'étudier les Indiens Suyá du Mato Grosso, au Brésil. J'ai l'impression que les ethnomusicologues choisissent la communauté qu'ils étudient pour une de ces trois raisons : certains, parce qu'ils aiment le son de la musique, d'autres, parce qu'ils aiment le pays ou la communauté (par expérience d'adulte ou pour y être nés), enfin un petit nombre choisissent comme moi leur site de recherche pour des raisons d'adéquation théorique. Profondément marqué par les œuvres de Max Weber, d'Emile Durkheim, de Karl Marx, de Claude Lévi-Strauss et de l'école britannique d'anthropologie sociale, je voulais analyser la relation entre la cosmologie, l'organisation sociale et la musique. Plus spécifiquement, je désirais faire des recherches sur la manière dont les gens organisent leur cosmos (concepts de temps, d'espace et de personne) et s'organisent en groupes (familles, moitiés,groupes cérémoniels), ainsi que sur la façon dont la musique s'accorde à la cosmologie et à la vie sociale. Ce sont là de vieilles questions posées par la philosophie et les sciences sociales, sauf pour la partie musicale. Etant donné que de nombreux scientifiques marxistes avaient déjà développé des théories sur la société capitaliste, je décidai d'étudier le sujet dans une société non capitaliste.

Bien entendu, j'avais aussi des motivations personnelles. Ayant toujours été lève-tôt, j'avais beaucoup de peine à rester éveillé toute la nuit. Je détestais me retrouver dans les grandes foules. Par conséquent, l'étude de la musique populaire dans les boîtes de nuit et les stades était exclue. J'aimais aussi l'idée des vastes espaces quasiment vides.

Mais une fois le problème et le mode de production non capitaliste choisis, il fallut envisager d'autres critères. Pour pouvoir étudier la relation réciproque entre ces différents aspects - croyances, vie sociale et musique - je devais analyser un groupe n'ayant pas été converti par les missionnaires (ce qui aurait changé sa cosmologie), ne faisant pas partie de la force de travail capitaliste (ce qui aurait changé ses relations sociales) et faisant de la musique. Seuls quelques rares endroits au monde correspondaient à ces critères : l'Australie, la Nouvelle Guinée et le bassin amazonien. Comme Judy était en train de préparer un doctorat en espagnol et en portugais, nous décidâmes d'aller au Brésil. Terence Turner me recommanda les Suyá qui, après avoir rencontré un explorateur allemand en 1884, refusèrent tout contact avec les Brésiliens jusque dans les années 1950 ; son choix me parut judicieux. Judy et moi, nous choisîmes les Suyá parce qu'il s'agissait d'une des rares sociétés septentrionales de langue Gê n'ayant pas encore été étudiée par le projet Harvard/Brésil central de David MayburyLewis. Des publications anciennes, parmi lesquelles les œuvres marquantes et fascinantes de Lévi-Strauss, aussi bien que des recherches plus récentes menées par les 
doctorants de Harvard, indiquaient la présence de longs cycles rituels chez tous les groupes septentrionaux de langue Gê. Les Gê étaient célèbres pour leur organisation sociale complexe, leur cosmologie étonnante et, pour ceux qui avaient lu Lévi-Strauss, leur mythologie ; mais personne n'avait encore jamais étudié leur musique. J’allais chez les Suyá sans avoir entendu plus de deux plages mal enregistrées de leur musique sur un disque Folkways. En fait, lorsque j'arrivai à Rio de Janeiro pour commencer mes recherches, je ne savais rien sur la musique Suyá et que peu sur le Brésil.

Ce que je découvris chez les Suyá semblait justifier la question originelle que je m'étais posée. A l'instar de nombreuses sociétés septentrionales de langue Gê, la cosmologie des Suyá est profondément dualiste - presque tout vient toujours par deux. Par exemple, au lieu d'avoir quatre directions, ils en ont deux : l'est et l'ouest (le début et la fin du ciel). A la place de quatre saisons, ils en ont deux : la saison sèche et la saison des pluies. De nombreux aspects de l'organisation sociale sont aussi arrangés par paires : les Suyá ont des moitiés cérémonielles très importantes, ainsi que d'autres divisions duales. Tout cela n'est pas inhabituel dans cette famille linguistique et dans cette région. On en trouve la description dans de nombreuses monographies, et Claude LéviStrauss en a parlé magistralement. Ce qui n'a cependant pas été observé, c'est que la musique suit exactement la même structure: la plupart des chants Suyá ont deux parties (le début et la fin). Chaque partie est structurée de façon identique, en "chercher le nom» et "dire le nom». Chaque vers a deux parties: le "texte référentiel » et les « syllabes du chant ». Parfois, la première partie, « début du chant ", est chantée face au côté oriental de la maison des hommes (l'est symbolisant le « début du ciel»), puis la seconde partie face au côté occidental (on s'adresse ainsi aux deux « extrémités »). 
Fig 2 : Tony Seeger dansant avec les Suyá.

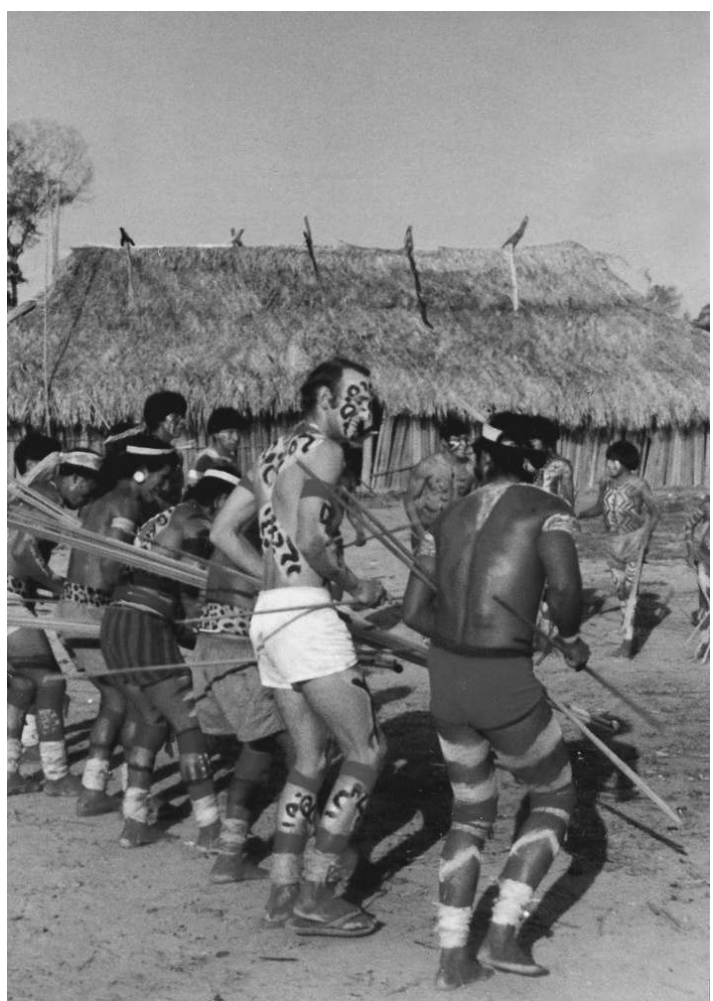

Photo : Judy Seeger, 1972

Je découvris que, non seulement, la structure de la plupart des chants Suyá individuels ou à l'unisson - est basée sur les mêmes principes que ceux qui régissent la cosmologie et l'organisation des institutions sociales, mais que la manière spécifique qu'a chaque personne de chanter reflète son âge et le statut de son sexe. Cela se fait de différentes façons très particulières. Les gens chantent ce qu'ils sont. Il existe, par exemple, un genre de chants individuels que seuls les garçons et les hommes chantent. Lorsque les garçons ont quatre ans environ, on leur apprend de très courtes mélodies, et leurs chants n'ont qu'une partie. A dix ans, ils commencent à chanter des chants complets à deux parties. A l'adolescence, on leur apprend à interpréter des chants composés de phrases musicales plus longues et plus nombreuses, qu'ils doivent chanter d'une voix haute et tendue. Lorsqu'ils deviennent pères, ils sont censés interpréter les mêmes chants mais dans un registre plus grave. Lorsqu'ils deviennent grands-pères, ils arrêtent complètement de chanter et n'émettent plus qu'un cri de fausset. Ces changements soniques sont tous reliés à l'évolution des cycles de la vie des garçons et des hommes. Les femmes restent dans leur maison natale, alors que, traditionnellement, les hommes la quittent pour résider pendant quelques années dans la maison des hommes, située au centre du village. Puis ils se marient et vont vivre avec leur femme dans une autre maison où ils élèvent leurs enfants.

Il serait trop long de développer ici ce sujet, qui a déjà été analysé dans Why Suyá Sing (2004 [1987c]). Les conclusions de la recherche suggèrent, d'une part, que la musique fait état des mêmes caractéristiques organisationnelles que le cosmos et la vie sociale, et, d'autre part, qu'elle façonne réellement la cosmologie et la vie sociale, du fait que 
ces dernières trouvent leur expression dans des cérémonies caractérisées par de musique et la danse.

Parfois je me demande si les Suyá et moi, nous ne nous sommes pas inconsciemment entendus à cause de notre amour respectif de la symétrie ! J'aimerais bien reprendre un projet d'étude comparative de la musique des Indiens Gê du Brésil, que j'avais l'intention d'entreprendre en 1988, lorsqu'au lieu d'aller au Brésil, je suis parti à Washington DC pour travailler à la Smithsonian. Ce projet vise à comparer les formes et les interprétations musicales de différents groupes Gê septentrionaux, et à déterminer de quelle manière leurs variations suivent celles de leur cosmologie et de leur organisation sociale.

Que pensez-vous avoir appris des Suyá?

En plus de ce que j'ai écrit dans mes livres, je dois dire que notre expérience des Indiens Suyá a profondément transformé ma vie, ainsi que celle de Judy et de nos enfants. Imaginez de vivre dans une société sans argent, où la nourriture est partagée entre un large réseau de parents, où la "richesse » est la connaissance et l'ornementation, où vous ne dites jamais "non » lorsqu'on vous demande quelque chose, où il n'y a ni police, ni armée, ni missionnaires, ni employeurs, ni usines. La nourriture arrive toute fraîche des jardins biologiques, des eaux claires de la rivière Suyá Missu, des forêts et des savanes apparemment sans limites. J'avais pris part aux manifestations de 1968 contre le bombardement du Cambodge; j'avais entendu parler de la brutalité de la dictature militaire au Brésil; et la société Suyá du début des années 1970 était totalement, mais totalement, différente. Cela ne veut pas dire qu'il n'y avait pas de pouvoir politique, d'inégalité, de peur ou de violence : il y en avait bien sûr. Mais c'était à une échelle très différente et en quelque sorte de façon moins apparente qu'aux EtatsUnis.

Maintenant, imaginez notre visite de leur point de vue : quelqu'un s'introduit chez vous, en disant « je suis anthropologue. Je viens habiter chez vous pour vous étudier pendant deux ans. Qu'avez-vous à dîner, ce soir? » Est-ce que vous le laisseriez entrer, sachant combien cela va vous coûter en argent, travail et perte d'intimité ? Les Suyá nous ont permis de rester, et nous leur resterons redevables d'une dette d'hospitalité jusqu'à la fin de notre vie.

Nous arrivâmes au village Suyá pendant la saison sèche de 1971, introduits par Claudio Villas-Boas, un des premiers Brésiliens à avoir établi un contact avec eux, vers 1959. Le gouvernement brésilien avait tellement tardé à nous livrer l'autorisation d'entrer dans leur territoire et le coût de la vie en ville était si élevé que ma bourse de recherche était épuisée. Sans argent depuis des mois, nous vivions de la généreuse hospitalité de quelques Brésiliens à qui nous avions été présentés à São Paulo. Nous étions installés chez les Suyá pour apprendre et non pour les vacciner, les instruire ou les « améliorer » comme le faisaient de nombreux Brésiliens ; c'est pourquoi nous ne leur faisions jamais de remarque sur ce qu'ils devaient faire ou ne pas faire. C'était en fin de compte la meilleure attitude à adopter. Les Suyá savaient par expérience que les non-Indiens étaient dirigistes. Une femme me dit un jour que les Suyá nous aimaient bien parce que nous ne leur donnions pas de conseils et que nous ne les critiquions pas. La raison pour laquelle je n'essayais jamais de donner des conseils aux Suyá, c'est qu'ils savaient tout, presque toujours mieux que moi. 
Là-dessus, j'avais raison; par contre, je me trompais sur les plaisanteries échangées entre les hommes brésiliens et suyá, caractéristiques de la plupart des relations interethniques. Croyant que les taquineries entre les non-Indiens et les Indiens de la région $=$ du Xingu étaient révélatrices de relations basées sur un statut hiérarchique, je ne blaguais pas avec eux ; ce qui leur faisait croire que j'étais toujours fâché contre eux, puisque je ne rigolais jamais.

Je n'avais pas compris que, lorsqu'il existe une relation à plaisanteries, le refus de plaisanter constitue une rupture de relation. La recherche sur le terrain est pleine de ces découvertes. Lorsque j'appris le Suyá, je me mis à blaguer. C'est amusant de plaisanter dans la langue que l'on est en train d'apprendre, en particulier pour une raison: lorsque les gens apprécient en riant, on est certain d'avoir dit quelque chose de juste. Au contraire, si les gens restent de marbre, on sait qu'on a raté son coup, la réaction étant immédiate et gratifiante. Les Suyá furent soulagés de voir que j'avais le sens de l'humour, même si je ne l'avais pas en portugais.

L'expérience de Judy fut très différente. Elle avait un bien meilleur sens de l'humour que moi. Les femmes suyá n'avaient jamais eu l'occasion de parler avec une femme non indienne parce qu'elles ne parlaient pas le portugais. Elles lui apprirent le Suyá pour pouvoir lui poser des questions sur tout ce qu'elles voulaient savoir depuis longtemps sur la société non indienne et surtout sur la vie des femmes. Judy arrêta d'écrire son journal après deux semaines, décidant de vivre comme tout le monde et non comme une anthropologue. Elle écrivit la chanson qui décrit un anthropologue, qui est gravée sur le bonus track du CD qui accompagne Why Suyá Sing,en se moquant gentiment de moi, avec beaucoup d'humour.

L'apprentissage de la langue suyá fut très difficile ; jusqu'ici personne ne l'avait encore étudiée. Nous prîmes un temps fou pour réussir à parler et, surtout, pour comprendre les réponses à nos questions générales. J'avais pris un cours sur l'apprentissage des langues non écrites, mais ce fut bien plus frustrant que ce que j'avais imaginé. Trois ou quatre jeunes gens parlaient un peu de portugais commercial, mais pas très couramment; les femmes et les anciens ne le parlaient pas du tout. Les Suyá nous enseignèrent un peu comme on apprend aux enfants, et nous saisîmes très vite le répertoire des réponses données à l'omniprésente question « Où vas-tu? ? : déféquer, me baigner, au jardin. J'écrivis d'interminables listes de sons dans un alphabet phonétique, certains étant des mots et d'autres des phrases. J'atteignis le stade où je pouvais poser des questions mais ne comprenais pas les réponses. L'enregistreur était alors bien pratique. Finalement, j'eus une conversation (la première d'un grand nombre) à propos de quelque chose d'abstrait: je posais une question, comprenais la réponse, posais une autre question et une conversation se développait. C'était au neuvième mois de notre séjour dans le village.

Il faut savoir que nous partagions une grande maison de chaume avec une trentaine de Suyá. Nous n'avions - par choix ou pour des raisons pratiques - pas de maison à nous, pas de table ni de chaises. Nous ne payions personne pour travailler pour nous, ce qui signifie que nous entendions parler la langue vingt-quatre heures sur vingt-quatre et que nous dialoguions constamment avec les Suyá. Lorsque nous nous retirions dans nos hamacs pour lire, ils se demandaient si nous étions tristes ou fâchés (c'était dans cet état seulement que nous arrêtions d'interagir avec le reste de la maison). Lorsque je ne sortais pas du village pour chasser ou pêcher en faisant de piteux - mais gratifiants - 
efforts, je tâchais de discuter avec ceux qui étaient restés. Souvent les hommes avec qui je voulais parler partaient à la chasse, à la pêche ou en voyage tandis que je restais au village. D'autres fois, ils étaient disponibles, mais j'étais trop épuisé par une journée de pêche et de chasse pour essayer de leur parler. C'était l'observation participante poussée à un degré inimaginable. En participant, j'apprenais ; en écoutant, j'entendais des choses que je n'aurais jamais pensé demander ; je garde la nostalgie de ces longues journées sur l'eau claire et scintillante de la rivière Suyá-Missu et de ma jeunesse passée là-bas en compagnie des Suyá.

Ils ont également une autre façon d'enseigner la langue à leurs enfants. Ils leur demandent de raconter quelque chose de très drôle ou d'obscène à une personne. Lorsque l'enfant - ou l'anthropologue - finit par comprendre ce qui a été dit, ils refusent de répéter. Puis arrive le tour d'une autre phrase ! Les hommes m'envoyaient à l'autre bout de la place pour dire des choses aux femmes et vice-versa. Tout le monde passait un moment agréable, bien qu'embarrassant. Beaucoup de ces mots, et les idées qu'ils exprimaient, furent essentiels pour mon travail ultérieur.

La recherche sur le terrain n'est facile nulle part. En trente-cinq ans, nous avons passé avec Judy plus de deux ans dans les villages suyá. Quinze mois de cette période furent consacrés à la recherche sur le terrain pour ma thèse, de 1971 à 1973. Ceux qui ont lu les premiers chapitres de mes livres (Seeger 1981 et 2004 [1987c]), savent combien ma recherche impliquait notre participation intensive à la vie quotidienne.

Les objets que nous leur apportions - les habituels perles de verroterie tchèques, miroirs, habits et couvertures - avaient leurs limites. Notre musique était le seul présent inépuisable. Par des nuits de clair de lune, les Suyá nous invitaient à venir faire de la musique et chanter au centre du village. Lorsque Judy me rejoignait sur la place, la plupart des femmes du village l'accompagnaient. Nous chantions «Michael Row the Boat Ashore» (« Michel arrive en ramant»), qui devenait dans leur langue «Quelque chose arrive en puant ». "The Gray Goose » racontait l'histoire de l'oie qu'on tuait et dont la chair coriace était immangeable. Et je chantais l'histoire du géant cannibale Abiyoyo que j'adaptais aux idées suyá sur les géants terrifiants et répugnants. A l'heure qu'il est, j'ai dû effrayer des générations d'enfants; en 2003 lorsqu'un enfant, épouvanté par l'histoire, se mit à crier, son père lui dit : «Ce n'est rien, moi aussi j'ai crié quand j'étais petit ». Nous échangions des chansons ; Judy chantait avec les femmes et moi je participais aux chants et danses des hommes. Les Suyá apprenaient nos chants exactement comme nous apprenions les leurs. Ce n'était un problème ni pour eux, ni pour nous. Ils connaissent les chants d'au moins six autres groupes indigènes en plus des leurs. Nos chansons n'étaient rien d'autre qu'un exemple de plus, agréable certes, mais pas particulièrement spécial, de la manière dont les "étrangers » font de la musique qu'ils peuvent s'approprier. 
Fig 3 : Tony et Judy Seeger sur la rivière Xingu (1972).

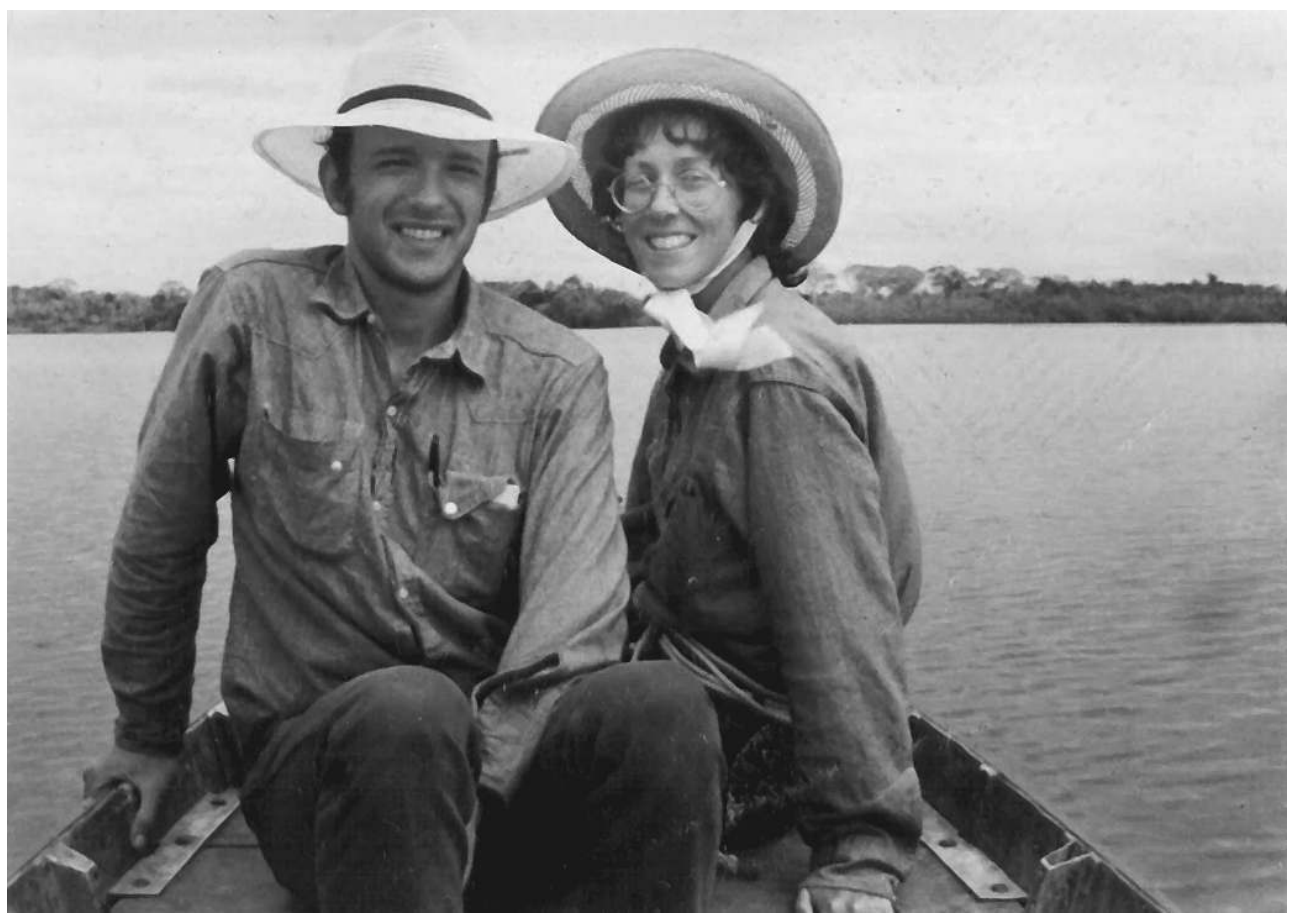

Après 1977, nous leur rendîmes des visites plus courtes, en général pendant la saison sèche où nos enfants risquaient moins d'attraper la malaria. Comme nous connaissions déjà la langue, nous n'avions plus besoin de rester longtemps sur place. Aujourd'hui, nous sommes capables d'atteindre le village en deux ou trois jours, après avoir atterri à Brasilia. A l'époque, cela prenait parfois des semaines pour arriver au village et près d'un mois pour en sortir.

La chose la plus mémorable que j'appris en accompagnant les Suyá dans les voyages en canoë, durant les années 1970, lorsque nous pagayions pendant des jours à remonter la rivière pour les chasses et les cérémonies collectives, fut de comprendre à quel $p=o i n t ~ l e$ voyage est important en soi. J'avais l'habitude de monter dans une voiture et rouler jusqu'à une destination. Lorsque les Suyá avaient une destination en tête, la chose importante à leurs yeux n'était pas uniquement l'endroit vers lequel ils se dirigeaient, mais toutes les occasions qui se présentaient pour pêcher, trouver du miel, s'amuser et vivre pleinement leur vie en y allant. Nous nous sentions privilégiés, Judy et moi, de pouvoir vivre avec les Suyá; vivre dans une société sans argent et sans aucun autre moyen habituel d'échange. Il est vrai que parfois le temps nous semblait long, parfois nous avions faim, parfois nous étions malades ; mais nous ne regrettions jamais d'y être allés.

Pourriez-vous nous parler des sujets clefs qui vous amenèrent à développer l'idée d'une anthropologie musicale?

Mon premier livre sur les Suyá était basé sur ma thèse de doctorat, à l'Université de Chicago. Intitulé Nature and Society in Central Brazil, the Suyá Indians of Mato Grosso, l'ouvrage était bâti sur le structuralisme de Lévi-Strauss et les idées du projet HarvardBrésil central élaboré par David Maybury-Lewis que j'ai déjà mentionné. Ce livre décrit comment des principes similaires organisent la cosmologie et l'organisation sociale du groupe. Je n'ai pas du tout parlé de musique dans ma thèse parce que, d'une part, je ne 
dominais pas suffisamment le sujet et, d'autre part, mon jury - Terence Turner, Victor Turner et Judith Shapiro - ne connaissait pas grand-chose à la musique, bien qu'ils fussent eux-mêmes des interprètes enthousiastes. Lorsque j'ai commencé à écrire Why Suyá Sing, au milieu des années 1980, j'ai relu le livre d'Alan Merriam sur l'anthropologie de la musique ${ }^{3}$. J'ai trouvé qu'une de ses faiblesses était une application trop directe de l' " anthropologie scientifique » à la musique. Pour marquer clairement la différence entre nos deux livres, j'ai parlé dans le mien d'une "anthropologie musicale ». En faisant cela, je créais un mouvement parallèle en anthropologie, vers des analyses plus dynamiques des processus sociaux.

Je commence toujours mes cours d'anthropologie musicale par des lectures de textes de Karl Marx, Max Weber et Emile Durkheim. Je pense que les grandes questions sur la nature de la société posées par les sciences sociales du XIX et du début du XX ${ }^{e}$ siècle sont vraiment intéressantes, profondément importantes et ne devraient pas être oubliées par les personnes qui s'occupent de musique. Les musicologues historiques se sont concentrés pendant des années sur les compositeurs et leurs œuvres; certaines études du folklore se sont longuement - et fort bien - penchées sur des textes et des produits culturels. L'anthropologie et les sciences sociales tâchent de poser des questions plus générales et considèrent que les arts sont insérés dans des processus sociaux complexes. C'est la raison pour laquelle j'estime que l'anthropologie est importante. Pourtant, elle a longtemps et systématiquement ignoré la musique. Selon moi, la musique a quelque chose à apprendre à l'anthropologie ; certains aspects de la musique peuvent éclairer les anthropologues sur les problèmes d'innovation, de créativité, d'interprétation, etc. J'avais écrit mon livre principalement pour les anthropologues. C'était la raison pour laquelle je l'avais abrégé (arrivé à la page 250 de mon manuscrit, j'éliminais une page chaque fois que j'en écrivais une nouvelle). Je suis sûr que beaucoup d'anthropologues ne l'ont même pas lu. Heureusement qu'il y eut assez d'ethnomusicologues pour épuiser la première édition de la Cambridge University Press. Après mon livre, un nombre impressionnant d'ouvrages ethnomusicologiques furent publiés. Beaucoup d'entre eux tournaient autour de la prise de décision individuelle, de la nature émergente de l'interprétation et d'autres sujets relatifs à ce que j'appellerai l'anthropologie musicale. 
Fig 4 : Danseurs de la cérémonie de souris, sujet de Why Suyá Sing, en train de chanter pendant la cérémonie d'imposition de nom (1996).

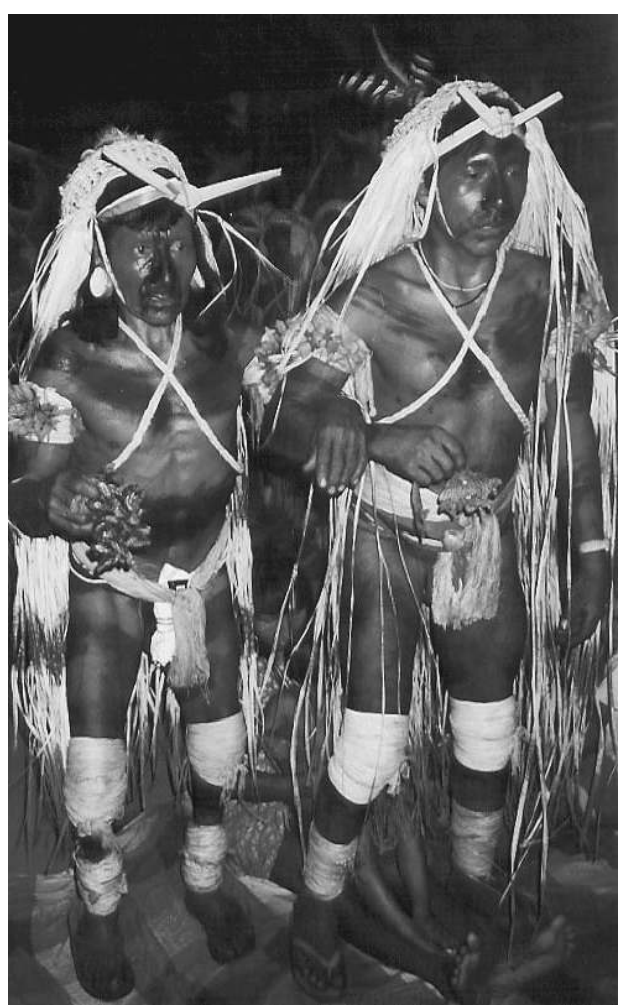

Comment a évolué votre relation avec les Suyá pendant ces décennies ?

Les Suyá m'appellent volontiers « notre anthropologue ». Ils ont l'impression de s'être mieux débrouillés avec nous que d'autres groupes indigènes voisins, dont l'anthropologue a disparu après une ou deux années, sans plus revenir ni leur fournir d'assistance financière. Lorsque ces Indiens leur demandent envieusement comment ils ont réussi à garder leur anthropologue pendant si longtemps, les Suyá répondent qu'ils nous ont mieux traités qu'eux. Et ils ont probablement raison !

Dans les années 1970, les Suyá m’interrogeaient sur la société brésilienne, mais ne semblaient pas convaincus par mes réponses. A la fin des années 1970, j'emmenai, sur sa demande, un des principaux dirigeants politiques du village avec une de ses femmes et deux de ses enfants à Rio de Janeiro, pour voir comment nous vivions. C'est une grande responsabilité de recevoir chez soi un de ses principaux «informateurs » (comme on les appelait à l'époque). Heureusement, nos enfants se sont bien entendus avec eux et nous nous sommes arrangés pour les repas, passant ainsi des moments agréables ensemble. Les visites du dirigeant politique à un match de foot ou à un des célèbres bidonvilles (favelas) de Rio, guidé par un ancien prisonnier, ont dû être souvent racontées au village. Ce genre d'expériences a approfondi, pendant des décennies, notre compréhension mutuelle.

Il y a toujours une question d'équité dans la recherche. Je me suis toujours assuré que les Suyá reçoivent toutes les redevances sur les disques que j'ai produits et au moins la moitié des droits d'auteur des livres que j'ai écrits. Je leur verse également une partie de la rémunération de mes conférences qui sont consacrées à eux. Lorsqu'ils ont un besoin communautaire urgent, ils savent qu'ils peuvent s'adresser à moi ; et, du fait que 
nous nous sentons profondément redevables de leur générosité, nous tâchons de les aider dans la mesure du possible, en récoltant des fonds auprès d'organismes de subvention ou en leur envoyant, lorsque nous le pouvons, une partie de nos propres économies. Nous tâchons de limiter ces donations à des choses dont la communauté entière peut bénéficier.

J'ai décrit dans la postface de la nouvelle édition de Why Suyá Sing comment les Suyá nous ont invités, en 1993, pour nous consulter sur des problèmes territoriaux auxquels ils étaient confrontés. Sans entrer dans le détail, je dirais que leur demande a ouvert une nouvelle ère dans nos relations. Nous ne les avions pas vus pendant douze ans; depuis 1994, nous leur rendons visite chaque année ou au moins tous les deux ans.

Pendant et après cette recherche, vous avez travaillé dans une université brésilienne. Pouvez vous dire quelques mots sur la façon dont vous avez considéré votre sujet et votre approche dans cet environnement professionnel ?

En 1970, lorsque je suis arrivé au Brésil pour ma recherche de thèse, Roberto Da Matta dirigeait le programme de doctorat en anthropologie sociale au Muséum national (qui faisait partie de l'Université fédérale de Rio de Janeiro). Je l'avais rencontré, en hiver 1968, au séminaire de Victor Turner, à l'Université Cornell. Il fut extrêmement serviable et généreux et supporta patiemment mon apprentissage laborieux de la langue. En 1973, lorsque je me préparais à rentrer aux Etats-Unis, il me proposa d'enseigner un cours avec lui. C'était une occasion rêvée; je gagnerais assez d'argent pour vivre pendant trois mois à Rio et cela permettait à Judy de retourner sur le terrain pour poser certaines questions dont les réponses me semblaient nécessaires pour la rédaction de certaines parties de ma thèse.

Nous eûmes énormément de plaisir à travailler ensemble, Roberto et moi, et j'appréciais la compagnie de mes collègues anthropologues brésiliens qui enseignaient dans le même programme. La plupart d'entre eux étaient depuis quelques années diplômés de différentes institutions dont l'anthropologie à São Paulo, Manchester, Paris et Cambridge, Massachusetts; la linguistique à l'Institut Patrice Lumumba de Moscou ; et les sciences politiques à l'Université de São Paulo. Leur approche de l'anthropologie était bien plus diversifiée qu'aux Etats-Unis. Ils aimaient nous entendre chanter et faire de la musique. Et rien ne valait une bière brésilienne bien fraîche par une journée chaude, à Rio de Janeiro !

En 1974, Roberto eut soudain la possibilité d'engager cinq professeurs adjoints à des postes fixes. Il choisit quatre scientifiques brésiliens, puis me téléphona aux Etats-Unis pour me proposer le cinquième poste : il s'agissait de donner un cours sur les Indiens du Brésil dans son institution. Lui-même travaillait à cette époque sur le carnaval et la société nationale brésilienne. Il convint avec ses collègues d'engager un spécialiste des Indiens brésiliens qui parlât une langue native et qui fût sympathique.

L'attrait de cet excellent département, la possibilité de continuer mes recherches, sans oublier la bière fraîche, furent irrésistibles. Judy et moi, nous retournâmes au Brésil. J'enseignai au Muséum national pendant sept merveilleuses années. Nos deux filles naquirent là bas et Judy put approfondir ses recherches sur le terrain, sur une tradition de romances chantées dans une communauté de pêcheurs à environ $600 \mathrm{~km}$ au nord de Rio de Janeiro. Elle développa le sujet dans une thèse de doctorat qu'elle publia plus tard sous la forme d'un livre (J.Seeger 1990). J'appris énormément de choses sur l'anthropologie brésilienne et sur la vie académique au Brésil. Interrogeant un de mes 
collègues sur la raison de mon élection à la présidence du département (un des plus grands département d'anthropologie d'Amérique du Sud, avec trente-trois professeurs), en 1980, il me répondit en riant que c'était pour me faire connaître le Brésil. Ils ont réussi !

L'anthropologie brésilienne se consacrait essentiellement à l'étude de la société brésilienne. La plupart de mes collègues s'occupaient de communautés rurales ou d'anthropologie urbaine. C'était diamétralement opposé à la démarche de l'Université de Chicago, consacrée à l'étude des communautés situées à l'extérieur des Etats-Unis. Le Brésil possède une forme très anthropologique d'autoréflexion - une bonne partie des idées populaires sur le pays ont été formulées par l'anthropologue Gilberto Freyre. C'est l'un des rares pays qui a eu à sa tête un spécialiste de sciences sociales (Fernando Henrique Cardoso est politologue et sa femme Ruth une très bonne anthropologue). Mes collègues s'adressaient par le biais d'importantes publications à l'intelligentsia urbaine et à la société en générale. Les journalistes recherchaient notre avis. Nos écrits paraissaient dans les journaux et nos commentaires dans des magasines du genre Time et Newsweek. Cet aspect de la vie intellectuelle brésilienne me plaisait beaucoup. Aux Etats-Unis, les universités se sont retirées, de gré ou de force, de l'arène politique et sociale. De nos jours, le réservoir de la pensée se trouve dans l'opinion publique plutôt que dans les universités de notre pays. Au Brésil, le réservoir de la pensée se trouvait à l'université. C'était là la source de la réflexion, des commentaires documentés, de la critique et, parfois, de l'activisme. Il y avait, parait-il, des espions du gouvernement dans le musée. Nous nous réunissions donc autour d'une fontaine pour parler de sujets que nous ne voulions pas divulguer.

De nombreux lecteurs connaissent sans doute votre rôle au sein des Archives de la musique traditionnelle (ATM), à l'Université d'Indiana. Pourriez-vous nous décrire cette partie de votre carrière?

Ma formation d'ethnomusicologue remonte à la période où j'occupai un poste de chercheur à l'Université d'Indiana, en 1980. J'y suivais les cours de Ruth Stone, de Ronald Smith et d'Alan Merriam (qui disparut dans un accident d'avion au milieu du semestre). Après avoir passé un été au Vermont, nous retournâmes au Brésil. Deux ans plus tard, le département d'anthropologie mit au concours le poste de Merriam; je posai ma candidature et fus engagé.

Nous avions des raisons personnelles pour quitter le Brésil. Nous pensions que nos enfants devaient apprendre l'anglais et mieux connaître leurs grands-parents (qui ne parlaient pas le portugais). Judy avait terminé ses recherches. Je commençais à plus écrire sur la musique Suyá, et il y avait plus d'ethnomusicologues à l'Université d'Indiana que dans tout le Brésil. L'inflation était terriblement élevée et mon salaire de fonctionnaire brésilien ne suffisait plus. J'avais peur de ne pas pouvoir voyager pour me rendre aux conférences ou voir nos familles. Bien que j'aie aimé tous les métiers que j'ai exercés pendant les décennies de ma carrière, mes années au Museu Nacional de Rio de Janeiro restent, pour moi, celles des plus grands défis et progrès intellectuels.

Je devins directeur des Archives de la musique traditionnelle (ATM) de l'Université d'Indiana par hasard. Lorsque je fus nommé professeur adjoint d'anthropologie à Indiana, le doyen de l'Ecole supérieure des arts et des sciences me demanda si je voulais me charger de la direction des archives. J'acceptai après avoir consulté mes collègues de l'université. 
J'avais quelques notions d'archivage. J'avais visité L'ATM en 1969, lorsque je préparais ma maîtrise à l'Université de Chicago. J'y avais déposé mes enregistrements de terrain à mon retour, en 1973. J'avais également suivi quelques cours d'archivage audiovisuel avec la directrice adjointe, Louise Spear. L'ATM avait besoin d'un directeur mais il avait un personnel très compétent. J'appris facilement ce que je ne savais pas grâce à la générosité du personnel de l'ATM, des archivistes et des ingénieurs du son de la Library of Congress, du British Institute of Recorded Sound, ainsi que des Phonogram-Archive de Berlin et de Vienne.

En tant que nouveau directeur, il me paraissait indispensable de déterminer, avant tout, si des archives étaient importantes ou non. Il était toujours possible de préférer jeter le vieux matériel et de dégager l'espace et les fonds pour créer de la nouvelle musique. Je décidai de commencer par l'hypothèse zéro, selon laquelle l'ATM ne méritait pas de continuer, et j'attendis la preuve du contraire. Tandis que j'étais dans cette phase, nous reçûmes une lettre des Indiens Fox qui souhaitaient exécuter une cérémonie dont ils avaient oublié certaines parties. Ils savaient qu'autrefois un anthropologue l'avait enregistrée et nous demandaient si ces bandes se trouvaient toujours à l'Université d'Indiana. Elles s'y trouvaient effectivement.

Je lisais à cette époque Le 18 Brumaire de Louis Bonaparte de Karl Marx. Il est écrit dans la première partie: "La tradition de toutes les générations mortes pèse comme un cauchemar sur le cerveau des vivants. Et, au moment précis où ils semblent occupés à se transformer eux-mêmes et à bouleverser la réalité, à créer l'absolument nouveau, c'est justement à ces époques de crise révolutionnaire qu'ils évoquent anxieusement et appellent à leur rescousse les mânes des ancêtres, qu'ils leur empruntent noms, mots d'ordre, costumes, afin de jouer la nouvelle pièce historique sous cet antique et vénérable travestissement et avec ce langage d'emprunt » (Marx 1994 [1852] : 437-438).

Marx critiquait cela en fonction des événements de 1848. Je retenais de cette observation le fait que les communautés considéraient leur passé comme faisant partie de leur processus de transformation. A mes yeux, les archives audiovisuelles renfermaient les traditions orales et les interprétations des membres des groupes subalternes dont la vie et l'histoire étaient généralement ignorées de l'histoire écrite des conquérants. Les collections d'archives pouvaient donc servir d'outil dans la lutte pour l'autodétermination des peuples du monde entier. Cela dotait les archives audiovisuelles d'une mission et d'un but qui dépassaient de loin leur fonction de simples dépositaires de collections destinées à la recherche. Il y avait là quelque chose que j'étais prêt à soutenir de tout mon cœur. C'est d'ailleurs ce qui continue à me faire croire que les archives sont si importantes.

Je réussis à prouver la valeur de l'ATM à l'Université, et obtins d'importantes subventions pour le transfert et la préservation des cylindres de cire et des fragiles disques d'acétate. L'année suivante, je pus obtenir des moyens d'archivage plus modernes et plus performants. J'avais l'impression que la survie des archives audiovisuelles dépendait uniquement d'un directeur qui soit à la fois un patron de cirque et un scientifique sérieux. Je me débrouillais assez bien en patron de cirque. Je signalais à tous les professeurs l'existence d'intéressantes collections dans leurs différentes spécialisations (par exemple, une collection de chansons grivoises italiennes). Un premier mai, je donnai même, avec Judy, un concert de chants 
syndicalistes devant un parterre composé du président de l'université, de collègues et d'étudiants.

Depuis que l'ethnomusicologie a délaissé le cabinet d'étude pour le terrain, les archives jouent un moindre rôle dans cette discipline. En même temps, celui-ci s'est accru pour les communautés qui ont été enregistrées. Cette évolution est due, d'une part, à une meilleure communication - les communautés savent où se trouvent les collections -, d'autre part, aux progrès technologiques qui permettent de faire des copies numériques impeccables et de les transférer. Les premiers enregistrements faits sur le terrain par des aventuriers ou des savants du XIX ${ }^{e}$ et du début du XX ${ }^{\mathrm{e}}$ siècles se sont révélés extrêmement importants pour les communautés enregistrées (Niles 2004); rappelezvous également mon expérience avec les Indiens Fox à l'ATM. Etonnamment, l'analyse scientifique de 1905 est moins importante que les produits dérivés de l'entreprise : les enregistrements eux-mêmes. En considérant notre propre travail dans cette perspective, nous arrivons à la conclusion que nous mettons l'accent sur les mauvais aspects de la recherche. Nos belles théories seront vite critiquées, et même discréditées. Nos enregistrements et nos notes de terrain, s'ils sont préservés, finiront à la longue par peser plus lourd que nos théories. Cela est vrai même lorsque nous étudions les sociétés qui se documentent elles-mêmes, comme un grand nombre d'entre elles le font aujourd'hui. Une partie importante des enregistrements faits dans des régions tropicales et humides ne survivront pas au-delà de quelques années s'ils ne sont pas déposés dans des archives spécialisées, capables de transférer les données d'un format à l'autre. Dans une centaine d'années, seuls les enregistrements archivés seront jouables. Si vous n'avez pas encore déposé vos enregistrements dans des archives, vous devez arrêter de lire ceci et vous mettre tout de suite au travail !

Après I'Université d'Indiana vous êtes parti à Washington DC pour diriger le Smithsonian Folkways. Pourriez-vous décrire cette partie de votre carrière?

Nous avions de grands projets pour 1988. Nous vivions depuis six ans à Bloomington, dans l'Indiana, et j'avais droit à un congé sabbatique. Je posai donc ma candidature pour l'obtention d'une bourse à la fondation Guggenheim en même temps qu'au National Endowment for the Humanities (NEH), afin de pouvoir retourner au Brésil et réaliser une étude comparative de la musique des sociétés de langue Gê du Brésil central. Nous avions assez d'argent et de congé pour passer deux ans au Brésil et nous faisions des plans pour un long séjour.

Pendant ce temps, Ralph Rinzler, qui était alors secrétaire adjoint à la Smithsonian Institution, essayait de me convaincre de devenir le premier conservateur et directeur d'une compagnie de disques que la Smithsonian était en passe d'acquérir : Folkways Records, ma maison de disques préférée. Ralph me recruta grâce à son assiduité, allant jusqu'à utiliser mon oncle Mike pour l'aider. Lors d'une de nos nombreuses réunions à Washington, mon oncle me dit combien la famille serait heureuse que j'accepte ce poste. Voyez un peu le schéma : je suis en train de planifier une chose, on m'en propose une autre et je ne peux pas résister. J'acceptai donc le poste à Folkways, en me souvenant de l'importance de certains de ses disques dans ma vie, les musique de l'Inde, d'Afrique et du Japon n'en constituant qu'une infime partie. Je voulus m'assurer qu'à la Smithsonian les disques continueraient à être utilisés, transformant la vie des gens de façon profonde et passionnante. J'étais convaincu de pouvoir accomplir ce travail mieux que quiconque. Né dans le milieu social et politique de la ville de New York, j'avais rencontré de nombreux artistes de Folkways; j'étais apparenté à certains des 
interprètes et des producteurs les plus prolifiques de cette maison ; je connaissais assez bien l'archivage pour pouvoir superviser l'organisation et la préservation de la collection. De plus, j'avais huit ans d'expérience administrative et étais un chercheur reconnu, avec quatre livres et de nombreuses autres publications : toutes choses qui importaient aux yeux de la Smithsonian. C'était franchement irrésistible. Nous avons donc renoncé à notre bourse de recherche, résilié notre congé sabbatique, pris un congé à l'Université d'Indiana - je démissionnai l'année suivante - et nous sommes partis nous installer à Washington DC au lieu d'aller au Brésil. Un tant soit peu fataliste, je me souvenais que mon grand-père Charles s'était rendu, à peu près à mon âge, à Washington pour se mettre au service du gouvernement. C'était peut-être un signe de mon destin génétique !

Je bénéficiais d'une grande latitude à la Smithsonian. J'étais engagé à la fois comme conservateur (savant qui s'occupe de science et non d'argent) et comme directeur (administrateur qui s'occupe d'argent sans être nécessairement un savant). J'étais libre de faire ce que je voulais, sauf de perdre de l'argent, et je devais mettre tous les titres de la collection à la disposition d'un large public. Il serait trop long de décrire ici les douze formidables années pendant lesquelles j'ai administré la collection Folkways; je laisse cela pour un autre article. J'ai déjà écrit une ou deux choses sur Folkways, notamment dans un article (Seeger 1996) où je décris quelques-uns des points les plus importants qu'un ethnomusicologue doit garder en mémoire lorsqu'il travaille avec les compagnies de disques.

Les ethnomusicologues doivent à tout prix se rendre compte que la musique est plus que du son, sur un plan pratique aussi bien que théorique. Lorsqu'ils s'apprêtent à faire des enregistrements, ils doivent obtenir les droits leur permettant d'utiliser ces sons enregistrés dans leurs thèses et leurs cours, de les publier ou de les archiver. Sans ces droits, les sons enregistrés sont virtuellement impropres à la diffusion. L'espoir de tirer de plus en plus de profit de la musique rend l'enregistrement sur le terrain plus difficile. Je soupçonne que cela remonte à l'énorme succès commercial de Graceland de Paul Simon et aux contrats faramineux signés avec Michael Jackson et d'autres musiciens très populaires. Des études sérieuses ont montré l'utilisation douteuse, souvent restée impayée, de musiques traditionnelles dans la musique de "fusion " (Zemp 1996; Guy 2002). Par ailleurs, de tels enregistrements sont importants pour l'avenir. Aujourd'hui, une partie des difficultés rencontrées par les ethnomusicologues vient du fait que beaucoup de gens transportent des enregistreurs mais que seuls un petit nombre d'entre eux est formé, au moins, pour respecter les problèmes éthiques concernant le processus de l'enregistrement.

Vous avez déménagé ensuite à UCLA, pour occuper un poste que vous avez cumulé, pendant quelques années, avec celui de Secrétaire général du Conseil international de la musique traditionnelle (ICTM). Pourriez-vous expliquer les raisons de votre retour à l'enseignement universitaire et parler de vos ambitions à UCLA ?

Après dix ans de conservation de la collection Folkways et de gestion des enregistrements de Smithsonian Folkways, je me mis à caresser l'idée de reprendre l'enseignement. J'avais l'impression de m'engourdir. A la Smithsonian, personne ne contestait mes idées comme le font les étudiants à l'université. En outre, j'étais sans cesse tourmenté par des problèmes liés à la production d'enregistrements, tels que les retards dans le calendrier et les difficultés à obtenir les droits; la comptabilité de la Smithsonian était très complexe et, au fur et à mesure que le personnel augmentait, 
cela devenait plus difficile de gérer et d'administrer l'opération sans perdre de l'argent. Nos enfants se préparaient à quitter la maison pour étudier à l'université. J'envisageai donc de quitter Washington DC pour trouver un travail ailleurs, si l'occasion se présentait. Pendant mes années à la Smithsonian, plusieurs institutions prestigieuses m'avaient généreusement proposé des postes; l'occasion de postuler à UCLA se présenta par hasard, en 1999. Je posai donc ma candidature au département d'ethnomusicologie, j'obtins le poste et j'avertis mon chef de la Smithsonian que plus rien ne me retenait désormais à Washington. Je ne quittai pourtant pas l'institution avant une année, car je voulais m'assurer que Folkways survivrait à la transition. La première transition après le fondateur est toujours difficile. J'eus la chance d'avoir pour successeur Daniel Sheehy qui a atteint sa sixième année avec succès. Je quittai donc la Smithsonian en été 2000 et occupai mon poste à UCLA dès l'automne.

Je trouvais un peu étrange d'intégrer le département dont mon grand-père Charles avait été un membre important dans les années 1960. Après l'avoir suivi à la trace à Washington DC, le suivre à Los Angeles relevait de la prédestination génétique. D'un autre côté, je ne voyais aucune raison valable de ne pas m'y rendre. J'aimais bien mes collègues. Ils étaient assez nombreux pour m'éviter d'endosser toute la charge de l'enseignement de l'ethnomusicologie (que je ne connaissais pas parfaitement). Par ailleurs, j'étais enchanté de donner des cours dans une branche que je n'avais encore jamais enseignée. J'avais toujours enseigné au département d'anthropologie, où je donnais des cours sur la parenté, le mariage, l'organisation sociale, la théorie anthropologique et, occasionnellement, l'ethnomusicologie. A UCLA, c'était différent : tous mes cours se rapportaient à la musique. Non seulement je recommençais à enseigner après une interruption de douze ans (mes étudiants en licence devaient avoir une dizaine d'années au moment où j'avais quitté l'enseignement), mais de plus, je commençais à enseigner un domaine dans lequel j'avais suivi, et donné, peu de cours. C'était formidable! Certains de mes étudiants étaient patients, d'autres critiques, et tous m'apportaient à lire des choses que je ne connaissais pas et m'apprenaient des choses auxquelles ne n'avais jamais pensé. Ils continuent d'ailleurs à le faire!

Et qu'en était-il de votre rôle au sein du Conseil international de la musique traditionnelle ?

Je ne sais pas si les gens m'ont appelé à siéger à la Society for Ethnomusicology (SEM) ou au Conseil international de la musique traditionnelle(ICTM) à cause de mon nom, de la qualité de mon travail, ou de mon prestige d'archiviste, d'auteur et de directeur de compagnie de disques, ou simplement parce que je disposais d'un budget de voyage. Peut-être aussi parce que je ne m'endors pas pendant les longues séances de réunion. Quelle qu'en soit la raison, j'ai été très actif dans les organisations professionnelles pendant la plus grande partie de ma vie, et je continue d'être membre d'un grand nombre d'entre elles. J'ai été membre du conseil, puis du conseil d'administration et finalement président de la SEM. De même, j'ai d'abord été élu conseiller administratif, puis président de l'ICTM (j'étais le seul nominé à la présidence, cette année-là). J’ai été membre du conseil d'administration de la North American Folk Music and Dance Alliance. J'ai été président du comité d'ethnomusicologie de l'Institut américain des études indiennes; je suis le président fondateur de la Research Archive Section de l'International Association of Sound and Audiovusual Archives (IASA). J'ai été membre de conseils consultatifs d'archives et j'ai tâché d'aider de nombreuses autres organisations.

Je devins membre du Conseil international de la musique traditionnellepour participer à une conférence mondiale qui avait lieu à New York, en 1981. J'avais projeté de 
renoncer à mon adhésion après la conférence. Vivant au Brésil avec un salaire académique brésilien, je ne croyais pas être en mesure de me rendre à d'autres réunions, étant donné que celles-ci se tenaient généralement loin du Brésil. Mais Nerthus et Dieter Christensen m'en dissuadèrent, et je restai membre. D'après moi, le rôle de l'ICTM est très différent de celui de la SEM. Il y a une plus grande diversité dans la formation et la provenance de ses membres, et j'ai toujours trouvé les conférences mondiales très stimulantes. Le fait que l'ICTM soit rigoureusement international et qu'il s'efforce de transcender les frontières politiques et géographiques est extrêmement important. Au cœur de l'ICTM, existent des groupes d'études - groupes de membres qui se réunissent pour discuter, faire des communications ou soutenir des publications sur un sujet spécifique. Parmi eux, on trouve des groupes d'études en ethnochoréologie, en étude de genre ou en anthropologie de la musique dans les cultures méditerranéennes. De plus, les colloques se proposent de réunir, dans un forum, les spécialistes de plusieurs pays pour aborder un seul thème à la fois. Récemment, un de ces colloques, intitulé «La discorde: identifier le conflit à l'intérieur de la musique; résoudre le conflit grâce à la musique », s'est réuni à l'Université de Limerick, en Irlande.

Je suis devenu secrétaire général de l'ICTM comme j'étais devenu directeur de l'ATM de l'Université d'Indiana. En 2001, lors de l'assemblée générale de la Conférence mondiale à Rio de Janeiro, le Secrétaire général, Dieter Christensen, annonça soudain sa démission, après vingt-et-un ans de service. J'étais président du programme de la conférence et dernier président sortant de l'ICTM. Par bonheur, mon président de département, Timothy Rice, et le doyen de l'Ecole des arts et de l'architecture de UCLA, Daniel Neuman, assistaient à la conférence. Avec leur concours, je pus arranger en quelques heures le déplacement du secrétariat de l'ICTM de l'Université de Columbia à UCLA, où il fut établi pendant quatre ans. Je n'avais pas l'intention d'accepter de responsabilités à UCLA; mais non seulement je réussis à trouver un soutien institutionnel sur la plage de Rio de Janeiro, mais de plus, du fait que je connaissais bien l'organisation pour l'avoir jadis présidée, je pus gérer tout cela. Grâce au financement du UCLA et de l'ICTM, j'engageai une excellente assistante, Kelly Salloum. A nous deux, nous réussîmes à refaire doucement fonctionner l'ICTM. Je compte parmi mes grandes réussites le passage en douceur du secrétariat du UCLA à l'Australian National University (ANU) à Canberra, avec Stephen Wild comme secrétaire général.

Parmi vos nombreuses publications, quelles sont celles que vous jugez les meilleures?

Chaque auteur a ses favoris, souvent des choses obscures qui sont lues par une poignée de gens. Les quatre que je choisirais pour ce genre de lecteurs traitent d'ornementation corporelle, de parfum et de musique.

Dans mon article de 1975, je suggère qu'il pourrait y avoir une relation systématique entre l'organe ornementé - l'oreille (l'ouie), la bouche (la parole/le chant), les yeux (la vision), le nez (l'odorat) - et les significations liées aux facultés qui lui sont associées. Je suggère aussi que chaque faculté est significative en soi, autant que dans son rapport avec les autres facultés (j'ai quelque chose d'un structuraliste déstructuré !). Du fait que les hommes et les femmes Suyá portent de grands disques aux oreilles et les hommes de grands disques labiaux, j'établis une relation avec l'importance de l'ouïe, de la parole et du chant (Seeger 1975). Le nez et les arômes sont très importants pour moi et pour les Suyá. 
Dans un congrès de parfumeurs et d'aromaticiens - où je fus interrompu par un homme déguisé en gorille qui giclait du parfum sur l'assistance au moyen d'une énorme banane - je fis mon exposé le plus général sur l'étude anthropologique des odeurs, dont il avait rarement été question jusque là (Seeger 1988a). Les anthropologues ont toujours eu tendance à ignorer la culture immatérielle, un domaine que se partagent la musique et les parfums. Why Suyá Sing est mon livre préféré. J'ai eu du plaisir à l'écrire et je l'ai intentionnellement abrégé, comme je vous l'ai déjà dit. Quant à mon article de 1996 sur les archives, la préservation et l'éthique, paru dans le Yearbook for Traditional Music, il aborde un certain nombre de sujets que j'ai traités dans divers écrits, au cours des dix dernières années.

Mais cette liste ne tient compte ni des quelques très bons projets de $\mathrm{CD}$ sur lesquels j'ai travaillé au Smithsonian Folkways Recordings, ni de mes contributions aux médias numériques, ni de certaines de mes notes qui bougèrent des choses que je croyais vouées à l'immobilité. Le "pire » des CD que j'ai réalisés à Folkways s'est vendu à plus d'exemplaires que tous mes livres réunis. Les pochettes de disque étaient capables d'atteindre et de transformer beaucoup plus de gens que mes articles de revues. J'aime toutes les contributions, petites et grandes, que j'ai faites pendant des années, aux projets de Folkways. Je trouve très important que les scientifiques écrivent dans différents formats pour toutes sortes d'audiences.

Fig 5 : Tony Seeger peint, assis en face de la maison des hommes, dans le village Suyá.

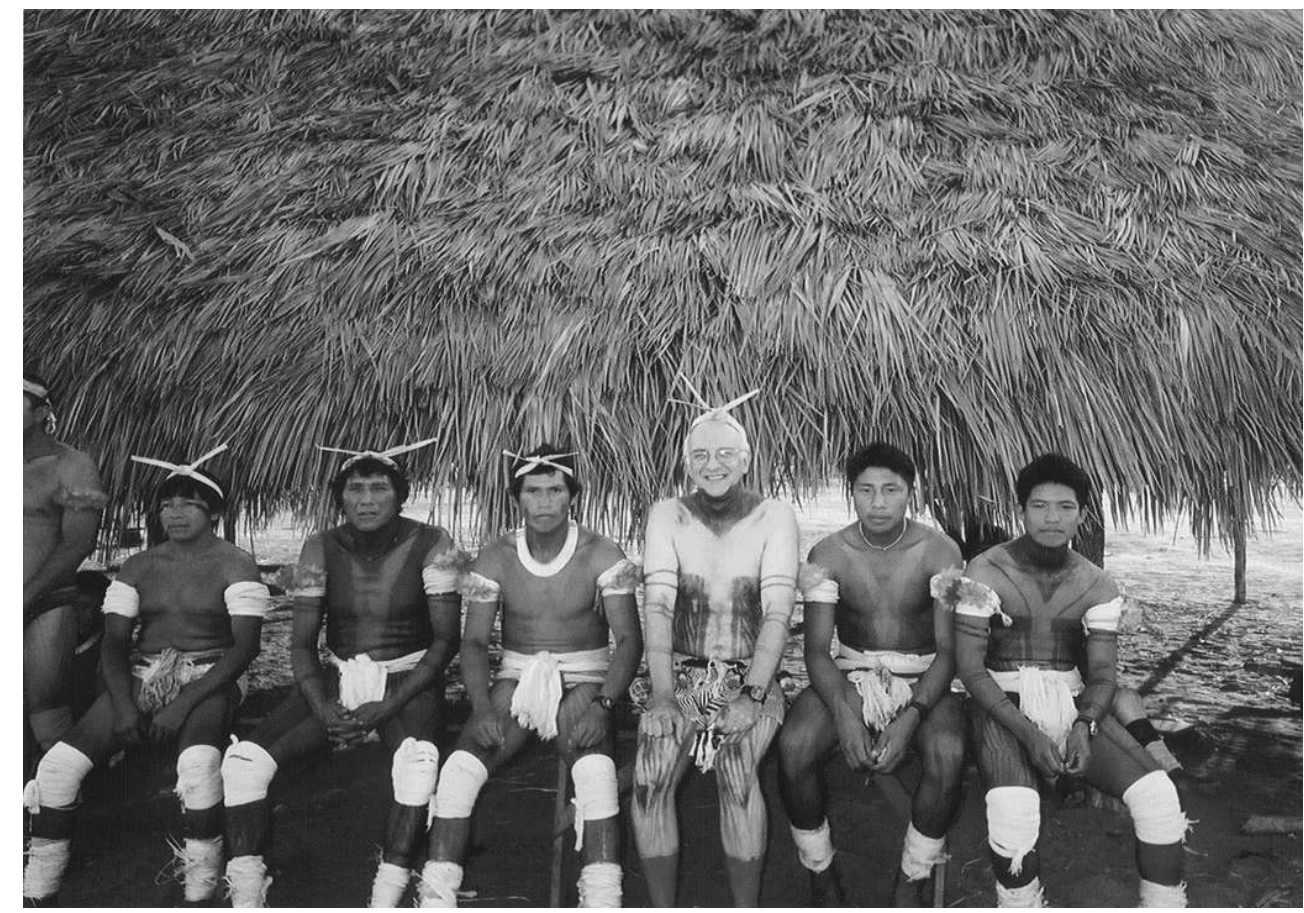

Photo : Hileia Seeger, 2003.

\section{Quels sont vos intérêts maintenant?}

Je commencerai un congé sabbatique en juillet 2007, mon premier depuis longtemps. Si tout va bien, je finirai un livre sur lequel je travaille depuis un moment sur la propriété musicale dans différentes sociétés, dont la nôtre. Mon livre ne cesse d'être retardé par un nombre croissant de publications sur la musique et les droits d'auteur. J'ai pourtant 
écrit beaucoup d'articles sur ce sujet depuis des années. Un autre projet que j'aimerais reprendre est l'étude comparative de la musique des sociétés de langue Gê. C'est le projet que j'aurais dû entreprendre si je n'étais pas allé à Washington DC plutôt qu'au Brésil. Il y a eu beaucoup plus de bonnes recherches sur la musique des peuples indigènes du Brésil, depuis 1988. Je pense qu'il est plus facile d'établir une base pour une étude comparative aujourd'hui qu'il y a deux décennies.

4 Pourtant, je ne fais qu'improviser. Qui sait ce qui pourrait encore arriver avant que je ne puisse à nouveau réaliser ces projets ?... En tout cas, une chose que les anthropologues feraient bien d'apprendre de la musique, c'est l'improvisation. Je n'ai pas planifié ma carrière ; j'ai accepté les choses intéressantes qui se sont présentées. Cela ne me serait jamais venu à l'esprit de diriger des archives, un label de disques ou l'ICTM. Il m'est arrivé beaucoup de choses sympathiques parce que je chantais et jouais du banjo. Nous sommes mariés, Judy et moi, depuis presque quarante ans, et nous sommes heureux de vivre ensemble et d'avoir deux merveilleuses filles qui sont maintenant des adultes. Lorsque deux universitaires se marient, leur vie professionnelle est un peu comme une danse où chacun essaie d'accommoder sa carrière à l'autre. Elever des enfants est aussi un peu comme une longue improvisation. Nous n'avons pas seulement besoin d'une anthropologie de la musique; nous devons également prendre des leçons de vie de la musique.

\section{BIBLIOGRAPHIE}

\section{Références}

GUY Nancy, 2002, « Trafficking in Taiwan Aboriginal Voices », in Sjoerd R. Jaarsma, ed. : Handle With Care :Ownership and Control of Ethnographic Materials. Pittsburgh : University of Pittsburgh Press : 195-209.

LORD Albert B., 2000, The Singer of Tales. Harvard : Harvard Studies in Comparative Literature. MARX Karl, 1994 [1852], Le 18 Brumaire de Louis Bonaparte. Paris : Gallimard, Bibliothèque de la Pléiade.

MERRIAM Alan P., 1964, The Anthropology of Music. Evanston, Ill : Northwestern University Press.

NILES Don, 2004, «Reclaiming the Past : The Value of Recordings to a National Cultural Heritage ", in Anthony Seeger and Shubha Chaudhuri, eds. : Archives for the Future: Global Perspectives on Audiovisual Archives in the 21st Century. Calcutta : Seagull Press : 196-206.

SEEGER Judith Leland, 1990, Count Claros : Study of a Ballad Tradition. New York : Garland.

ZEMP Hugo, 1996, «The/An Ethnomusicologist and the Record Business ». Yearbook for Traditional Music $28: 36-56$. 


\section{Bibliographie sélective d'Anthony Seeger}

SEEGER Anthony, 1975« The Meaning of Suyá Body Ornaments, a Suyá Example ». Ethnology 14 (no. S3) : 211-24.

SEEGER Anthony, 1977a, « Porque os índios Suyá cantam para as suas irmãs ? », in G.Velho, ed. : Arte e Sociedade, Ensaios de sociologia de arte. Rio de Janeiro : Zaha : 39-63.

SEEGER Anthony, 1977b, « Fixed Points on Arcs in Circles : The Temporal, Processual Aspects of Suyá Space and Society ", in Actes du XLII Congrés International des Americanistes, Paris, 2-9 Septembre 1976, ii : 330-42.

SEEGER Anthony, 1979, « What Can We Learn When They Sing ? Vocal Genres of the Suyá Indians of Central Brazil. » Ethnomusicology 23 : 373-94.

SEEGER Anthony, 1980a, Os Índios e Nos; Estudos sobre sociedades tribais brasileiras. Rio de Janeiro : Editora Campus.

SEEGER Anthony, 1980b, « Sing For Your Sister : The Structure and Performance of Suyá Akia », in N. McLeod and M. Herndon, eds. : The Ethnography of Musical Performance. Norwood PA : Norwood Editions : 7-43.

SEEGER Anthony, 1981, Nature and Society in Central Brazil : The Suyá Indians of Mato Grosso. Cambridge, MA : Harvard University Press.

SEEGER Anthony, 1982, Música Indigena : A arte vocal dos Suyá. Co-produced with members of the Suyá Indian Community. 12-inch disc with liner notes. São João del Rei : Edições Tacape 007, serie ethnomusicologia. [Reissued with revised notes on CD as volume 75 of Music of the Earth: Fieldworkers' Sound Collections. Tokyo : JVC Video Software Division 1992.]

SEEGER Anthony, 1985, Review Essay on general articles on ethnomusicology and related disciplines in the New Grove Dictionary of Music. Ethnomusicology 29 : 345-51.

SEEGER Anthony, 1986a, "Oratory is Spoken, Myth is Told, and Song is Sung, But They Are All Music To My Ears ", in J. Sherzer and G. Urban, eds. : Native South American Discourse. Berlin : Mouton de Gruyter : 59-82.

SEEGER Anthony, 1986b, « The Role of Sound Archives in Ethnomusicology Today. » Ethnomusicology 30 : 261-76.

SEEGER Anthony, 1987a, Early Field Recordings : A Catalogue of the Cylinder Collections at the Indiana University Archives of Traditional Music. Bloomington : Indiana University Press. [Edited jointly with Louise M. Spear.]

SEEGER Anthony, 1987b, « Music, Dance, and Drama : An Anthropological Perspective », in Jack Hopkins, ed. : Latin America : Perspectives on a Region. New York : Holmes \& Meier : 201-15. [with Anya P. Royce]

SEEGER Anthony, 1987c [2004], Why Suyá Sing : A Musical Anthropology of an Amazonian People. Cambridge : Cambridge University Press. [Re-issued with CD and new afterword in 2004 by University of Illinois Press]

SEEGER Anthony, 1988a, « Anthropology and Odor : From Manhattan to Mato Grosso. » Perfumer and Flavorist $13 / 4: 41-48$.

SEEGER Anthony, 1988b, « Voices, Flutes, and Shamans in Brazil. » The World of Music 30/2 : 22-39. 
SEEGER Anthony, 1989, « Dualism : Fuzzy Thinking or Fuzzy Sets ?», in D. Maybury-Lewis and U. Almagor, eds. : The Attraction of Opposites : Thought and Society in a Dualistic Mode. Ann Arbor : University of Michigan Press : 191-208.

SEEGER Anthony, 1990a, « Musics of Struggle », in P. Seitel, ed. : Program Book, 1990 Festival of American Folklife. Washington, DC : Smithsonian Institution : 56-69.

SEEGER Anthony, 1991a, « After the Alligator Swallows Your Microphone : The Future ( ?) of Field Recordings ", in N. C. McIntire, senior ed. : Discourse in Ethnomusicology III : Essays in Honor of Frank J. Gillis. Bloomington, Indiana : Ethnomusicology Publications Group : 37-49.

SEEGER Anthony, 1991b, « Creating and Confronting Cultures : Issues of Editing and Selection in Records and Videotapes of Musical Performances", in M. P. Baumann, ed. : Music in the Dialogue of Cultures : Traditional Music and Cultural Policy. Wilhelmshaven : Florian Noetzel Verlag : 290-302.

SEEGER Anthony, 1991c, « Styles of Musical Ethnography », in B. Nettl and P. Bohlman, eds. : Comparative Musicology and Anthropology of Music : Essays in the History of Ethnomusicology. Chicago : University of Chicago Press : 342-55.

SEEGER Anthony, 1991d, « When Music Makes History ", in S. Blum, P. Bohlman, and D. Neuman, eds. : Ethnomusicology and Modern Music History. Urbana : University of Illinois Press : 23-34.

SEEGER Anthony, 1992a, « Ethnography of Music », in H. Myers, ed. : Ethnomusicology : An Introduction. New Grove Handbook in Music. London : Macmillan : 88-109.

SEEGER Anthony, 1992b, « Ethnomusicology and Music Law. » Ethnomusicology 36 : 345-60.

SEEGER Anthony, 1992c, « Performance and Identity : Problems and Perspectives », in C. Robertson, ed. : Musical Repercussions of 1492. Washington, DC : Smithsonian Institution Press : 451-62.

SEEGER Anthony, 1996, « Ethnomusicologists, Archives, Professional Organizations, and the Shifting Ethics of Intellectual Property. » Yearbook for Traditional Music 28 : 87-105.

SEEGER Anthony, 1998a, « Musical Genres and Contexts », « Social Structure, Musicians, and Behavior », and " Musical Dynamics », in D. A. Olsen and D. E. Sheehy, eds. : Garland Encyclopedia of World Music Volume 2 : South America, Mexico, Central America and the Caribbean. New York : Garland Publishing Company : 43-53, 54-65, \& 66-78.

SEEGER Anthony, 1998b, Produced and presented a series of six 30-minute radio shows for BBC Radio on American Traditional Music. Aired in June-July 1998.

SEEGER Anthony, 2001a, " Americas », in The New Grove Dictionary of Music and Musicians, Second Edition. London : Macmillan, i : 469-82.

SEEGER Anthony, 2001b, «Intellectual Property and Audio Visual Archives and Collections », in Folk Heritage Collections in Crisis, Washington, DC : Council on Library and Information Resources : 36-47.

SEEGER Anthony, 2001c, «Technology and Media » (parts i-iii), in E. Koskoff, ed. : The Garland Encylclopedia of World Music : The United States and Canada. New York : Garland Publishing Company : 235-49.

SEEGER Anthony, 2003, «A Tropical Meditation on Comparison in Ethnomusicology : A Metaphoric Knife, a Real Banana, and an Edible Demonstration. " Yearbook for Traditional Music $34: 187-92$

SEEGER Anthony, 2004, Archives for the Future. Global Perspectives on Audiovisual Archives in the 21st Century. (Anthony Seeger \& Shubha Chaudhuri, eds.). Calcutta (India) : Seagull Books. 
Voir aussi : <http://www.ethnomusic.ucla.edu/people/seegercv.htm>

\section{NOTES}

1. Traduction de l'anglais par Ramèche Goharian.

2. Voir à ce propos l'entretien d'Isabelle Schulte-Tenckhoff avec Michael Asch, le fils de Moses ( Cahiers de musiques traditionnelles 16/2002 : 189-202) (ndlr).

3. Merriam 1964.

\section{AUTEURS}

\section{JONATHAN P. J. STOCK}

Jonathan P. J. Stockest professeur d'ethnomusicologie à l'Université de Sheffield. Il a consacré l'essentiel de ses recherches à la musique de Chine et de Taiwan; mais il a également écrit sur l'analyse musicale, la scène de la musique folk anglaise et les concertos pour piano de Mozart. Son plus récent livre, Huju : Traditional Opera in Mordern Shanghai (Oxford, 2003), est en train d'être traduit en chinois pour être publié. J. Stock est également co-éditeur de la revue The World of Music. 